\title{
Specimen records for North American Lepidoptera (Insecta) in the Oregon State Arthropod Collection. Lycaenidae Leach, 1815 and Riodinidae Grote, 1895
}

Jon H. Shepard

Paul C. Hammond

Christopher J. Marshall

Oregon State Arthropod Collection, Department of Integrative Biology, Oregon State University, Corvallis OR 97331

Cite this work, including the attached dataset, as:

Shepard, J. S, P. C. Hammond, C. J. Marshall. 2019. Specimen records for North American Lepidoptera (Insecta) in the Oregon State Arthropod Collection. Lycaenidae Leach, 1815 and Riodinidae Grote, 1895. Catalog: Oregon State Arthropod Collection 3(2). (beta version). http:// dx.doi.org/10.5399/osu/cat osac.3.2.4594

\section{Introduction}

These records were generated using funds from the LepNet project (Seltmann) - a national effort to create digital records for North American Lepidoptera. The dataset published herein contains the label data for all North American specimens of Lycaenidae and Riodinidae residing at the Oregon State Arthropod Collection as of March 2019. A beta version of these data records will be made available on the OSAC server (http: / / osac.oregonstate.edu/IPT) at the time of this publication. The beta version will be replaced in the near future with an official release (version 1.0), which will be archived as a supplemental file to this paper.

\section{Methods}

Basic digitization protocols and metadata standards can be found in (Shepard et al. 2018). Identifications were confirmed by Jon Shepard and Paul Hammond prior to digitization. Nomenclature follows that of (Pelham 2008).

\section{Results}

The holdings in these two families are extensive. Combined, they make up 25,743 specimens $(24,598$ Lycanidae and 1145 Riodinidae). While the majority of specimens are from the Pacific Northwest, the collection possesses examples of species from throughout North America.

Within the Lycaenidae, the collection holds 316 distinct species-level taxa (species and supspecies), classified into the following genera: Atlides Hübner, 1819, Brephidium Scudder, 1876, Callophrys Billberg, 1820, Calycopis Scudder, 1876, Celastrina Tutt, 1906, Chlorostrymon Clench, 1961, Cupido Schrank, 1801, Cyanophrys Clench, 1961, Cyclargus Nabokov, 1945, Echinargus Nabokov, 1945, Electrostrymon Clench, 1961, Erora Scudder, 1872, Euphilotes Mattoni, 1978, Glaucopsyche Scudder, 1872, Hemiargus Hübner, 1818, Lampides Hübner, 1819, Leptotes Scudder, 1857, Ministrymon Clench, 1961, Parrhasius Hübner, 1819, Philotes Scudder, 1876, Philotiella Mattoni, 1978, Plebejus Kluk, 1780, Rekoa Kaye, 1904, Strymon Hübner, 1818, Tmolus Hübner, 1819, Vaga Zimmerman, 1958, Zizula Chapman, 
1910 (Table 1).

The Riodinidae are less extensive, with onlh 20 species-level taxa classified into the following genera: Apodemia C. Felder \& R. Felder, 1865, Calephelis Grote \& Robinson, 1869, Caria Hübner, 1823, Emesis Fabricius, 1807, Lasaia H. Bates, 1868, (Table 2).

Table 1.

Taxon \# specimens

\begin{tabular}{|c|c|}
\hline Atlides halesus corcorani Clench, 1942 & 115 \\
\hline Atlides halesus halesus (Cramer, 1777) & 33 \\
\hline Brephidium exilis (Boisduval, 1852) & 142 \\
\hline Brephidium pseudofea (Morrison, 1873) & 22 \\
\hline Callophrys affinis undetermined sspp. & 67 \\
\hline Callophrys affinis affinis(Edwards, 1862) & 5 \\
\hline Callophrys affinis apama (Edwards, 1882) & 30 \\
\hline Callophrys affinis homoperplexa Barnes \& Benjamin, 1923 & 42 \\
\hline Callophrys affinis washingtonia Clench, 1944 & 23 \\
\hline Callophrys augustinus annetteae (Passos, 1943) & 19 \\
\hline Callophrys augustinus augustinus (Westwood, 1852) & 23 \\
\hline Callophrys augustinus croesioides (Scudder, 1876) & 30 \\
\hline Callophrys augustinus iroides (Boisduval, 1852) & 380 \\
\hline Callophrys dumetorum undetermined sspp & 46 \\
\hline Callophrys dumetorum dumetorum & 411 \\
\hline Callophrys dumetorum oregonensis Górelick, 1968 & 171 \\
\hline Callophrys eryphon eryphon (Boisduval, 1852) & 343 \\
\hline Callophrys eryphon fusca (Austin, 1998) & 5 \\
\hline Callophrys eryphon pallescens (Austin, 1998) & 6 \\
\hline Callophrys eryphon sheltonensis (Chermock \& Frechin, 1949) & 64 \\
\hline Callophrys fotis undetermined sspp. & 57 \\
\hline Callophrys gryneus castalis (Edwards, 1871) & 36 \\
\hline Callophrys gryneus chalcosiva Clench, 1981 & 353 \\
\hline Callophrys gryneus gryneus (Hübner, 1819) & 70 \\
\hline Callophrys gryneus juniperaria (Comstock, 1925) & 24 \\
\hline Callophrys gryneus loki (Skinner, 1907) & 46 \\
\hline Callophrys gryneus mansfieldi (Tilden, 1951) & 9 \\
\hline Callophrys gryneus nelsoni (Boisduval, 1869) & 193 \\
\hline Callophrys gryneus plicataria Johnson, 1976 & 77 \\
\hline Callophrys gryneus rosneri Johnson, 1976 & 3 \\
\hline Callophrys gryneus siva (Edwards, 1874) & 79 \\
\hline Callophrys henrici henrici (Grote \& Robinson, 1867) & 35 \\
\hline
\end{tabular}


Table 1 (continued).

Taxon

\#specimens

\begin{tabular}{|c|c|}
\hline Callophrys henrici margaretae (dos Passos, 1943) & 4 \\
\hline Callophrys henrici solatus (Cook \& Watson, 1909) & 12 \\
\hline Callophrys henrici turneri (Clench, 1943) & 4 \\
\hline Callophrys hesseli (Rawson \& Ziegler, 1950) & 7 \\
\hline Callophrys irus arsace (Boisduval \& LeConte, 1835) & 11 \\
\hline Callophrys irus hadros (Cook \& Watson, 1907) & 41 \\
\hline Callophrys johnsoni (Skinner, 1904) & 88 \\
\hline Callophrys lanoraieensis (Sheppard, 1934) & 35 \\
\hline Callophrys mcfarlandi Ehrlich \& Clench, 1960 & 15 \\
\hline Callophrys mossii bayensis Brown, 1969 & 44 \\
\hline Callophrys mossii hidakupa (Emmel, Emmel \& Mattoon, 1998) & 1 \\
\hline Callophrys mossii mossii (Edwards, 1881) & 118 \\
\hline Callophrys mossii schryveri (Cross, 1937) & 200 \\
\hline Callophrys mossii windi (Clench, 1943) & 77 \\
\hline Callophrys muiri (Edwards, 1881) & 54 \\
\hline Callophrys niphon (Hübner, 1819) & 13 \\
\hline Callophrys niphon clarki (Freeman, 1938) & 58 \\
\hline Callophrys polios maritima (Emmel, Emmel \& Mattoon, 1998) & 36 \\
\hline Callophrys polios obscurus Ferris \& Fisher, 1973 & 194 \\
\hline Callophrys polios polios (Cook \& Watson, 1907) & 148 \\
\hline Callophrys sheridanii interrupta Austin, 1998 & 48 \\
\hline Callophrys sheridanii lemberti Tilden, 1963 & 157 \\
\hline Callophrys sheridanii newcomeri Clench, 1963 & 245 \\
\hline Callophrys sheridanii paradoxa Scott, 1986 & 2 \\
\hline Callophrys sheridanii pseudodumetorum Emmel, Emmel \& Mattoon, 1998 & 190 \\
\hline Callophrys sheridanii sheridanii (Edwards, 1877) & 71 \\
\hline Callophrys spinetorum spinetorum (Hewitson, 1867) & 231 \\
\hline Callophrys xami xami (Reakirt, 1867) & 11 \\
\hline Calycopis cecrops (Fabricius, 1793) & 61 \\
\hline Calycopis isobeon (Butler \& Druce, 1872) & 58 \\
\hline Celastrina echo cinerea (Edwards, 1883) & 44 \\
\hline Celastrina echo echo (Edwards, 1864) & 33 \\
\hline Celastrina echo nigrescens (Fletcher, 1903) & 585 \\
\hline Celastrina echo sidara (Clench, 1944) & 13 \\
\hline Celastrina idella Wright \& Pavulaan, 1999 & 3 \\
\hline Celastrina ladon (Cramer, 1780) & 25 \\
\hline
\end{tabular}


Table 1 (continued).

Taxon

\#specimens

Celastrina lucia (Kirby, 1837)

Celastrina neglecta (Edwards, 1862)

Celastrina neglectamajor Opler \& Krizek, 1984

Celastrina nigra (Forbes, 1960)

Chlorostrymon simaethis (Drury, 1773)

65

75

Cupido amyntula albrighti (Clench, 1944)

Cupido amyntula amyntula (Boisduval, 1852)

270

Cupido amyntula herri (Grinnell, 1901)

Cupido amyntula montanorum (Austin, 1998)

Cupido amyntula valeriae (Clench, 1944)

Cupido comyntas comyntas (Godart, 1824)

Cupido comyntas sissona (Wright, 1905)

Cupido comyntas texana (Chermock, 1945)

1

1

7

31

11

270

Cyanophrys goodsoni (Clench, 1946)

Cyanophrys miserabilis (Clench, 1946)

Cyclargus thomasi bethunebakeri (Comstock \& Huntington, 1943)

Echinargus isola (Reakirt, 1866)

Electrostrymon angelia angelia (Hewitson, 1874)

Erora laeta (Edwards, 1862)

Erora quaderna sanfordi Passos, 1940

16

27

10

63

82

24

32

11

36

112

15

18

Eumaeus atala (Poey, 1832)

22

Euphilotes battoides undetermined sspp (Behr, 1867)

19

Euphilotes battoides battoides (Behr, 1867) 67

Euphilotes battoides fusimaculata Austin, 1998

Euphilotes battoides mazourka Pratt \& Emmel, 1998

Euphilotes battoides vernalis Pratt \& Emmel, 1998

Euphilotes bernardino (Barnes \& McDunnough, 1916)

Euphilotes centralis centralis (Barnes \& McDunnough, 1917)

Euphilotes centralis hadrochilus Pratt \& Emmel, 1998

Euphilotes enoptes undetermined sspp. 33

3

Euphilotes enoptes ancilla (Barnes \& McDunnough, 1918)

Euphilotes enoptes bayensis (Langston, 1964)

Euphilotes enoptes columbiae (Mattoni, 1954)

Euphilotes enoptes dammersi (Comstock \& Henne, 1933)

Euphilotes enoptes enoptes (Boisduval, 1852)

Euphilotes enoptes langstoni (Shields, 1975)

(see discussion)

13

366

99

11

13

13


Table 1 (continued).

Taxon

\#specimens

\begin{tabular}{|c|c|}
\hline Euphilotes enoptes tildeni (Langston, 1964) & 23 \\
\hline Euphilotes glaucon undetermined sspp. & 63 \\
\hline Euphilotes glaucon comstocki (Shields, 1975) & 3 \\
\hline Euphilotes glaucon glaucon (Edwards, 1871) & 159 \\
\hline Euphilotes glaucon oregonensis (Barnes \& McDunnough, 1917) & 321 \\
\hline Euphilotes mojave mojave (Watson \& Comstock, 1920) & 11 \\
\hline Euphilotes pallescens arenamontana Austin, 1998 & 7 \\
\hline Euphilotes pallescens calneva Emmel \& Emmel, 1998 & 4 \\
\hline Euphilotes pallescens confusa Pratt \& Emmel, 1998 & 5 \\
\hline Euphilotes pallescens mattonii (Shields, 1975) & 2 \\
\hline Euphilotes pallescens ricei Austin, 1998 & 65 \\
\hline Euphilotes rita undetermined sspp. & 3 \\
\hline Euphilotes rita coloradensis (Mattoni, 1966) & 15 \\
\hline Euphilotes spaldingi (Barnes \& McDunnough, 1917) & 14 \\
\hline Feniseca tarquinius tarquinius (Fabricius, 1793) & 42 \\
\hline Glaucopsyche lygdamus arizonensis McDunnough, 1936 & 14 \\
\hline Glaucopsyche lygdamus australis Grinnell, 1917 & 59 \\
\hline Glaucopsyche lygdamus columbia (Skinner, 1917) & 561 \\
\hline Glaucopsyche lygdamus couperi Grote, 1873 & 53 \\
\hline Glaucopsyche lygdamus deserticola Austin \& Emmel, 1998 & 13 \\
\hline Glaucopsyche lygdamus incognitus Tilden, 1974 & 55 \\
\hline Glaucopsyche lygdamus jacki Stallings \& Turner, 1947 & 52 \\
\hline Glaucopsyche lygdamus lygdamus (Doubleday, 1842) & 4 \\
\hline Glaucopsyche lygdamus mildredae Chermock, 1944 & 5 \\
\hline Glaucopsyche lygdamus orcus (Edwards, 1869) & 31 \\
\hline Glaucopsyche lygdamus xerces (Boisduval, 1852) & 15 \\
\hline Glaucopsyche piasus (Boisduval, 1852) & 279 \\
\hline Habrodais grunus undetermined sspp. & 174 \\
\hline Habrodais grunus grunus (Boisduval, 1852) & 11 \\
\hline Habrodais grunus herri Field, 1938 & 131 \\
\hline Habrodais grunus lorquini Field, 1938 & 103 \\
\hline Hemiargus ceraunus undetermined sspp. & 25 \\
\hline Hemiargus ceraunus antibubastus Hübner, 1818 & 16 \\
\hline Hemiargus ceraunus astenidas (Lucas, 1857) & 27 \\
\hline Hemiargus ceraunus gyas (Edwards, 1871) & 14 \\
\hline Hypaurotis crysalus undetermined sspp. & 140 \\
\hline
\end{tabular}


Table 1 (continued).

Taxon

\# specimens

\begin{tabular}{|c|c|}
\hline Lampides boeticus (Linnaeus, 1767) & 48 \\
\hline Leptotes cassius cassidula (Boisduval, 1870) & 10 \\
\hline Leptotes cassius theonus (Lucas, 1857) & 49 \\
\hline Leptotes marina (Reakirt, 1868) & 114 \\
\hline Lycaena arota arota & 199 \\
\hline Lycaena arota nubila (Comstock, 1926) & 43 \\
\hline Lycaena arota schellbachi (Tilden, 1955) & 25 \\
\hline Lycaena arota virginiensis (Edwards, 1870) & 13 \\
\hline Lycaena cupreus undetermined sspp & 13 \\
\hline Lycaena cupreus cupreus (Edwards, 1870) & 100 \\
\hline Lycaena cupreus lapidicola Emmel \& Pratt, 1998 & 73 \\
\hline Lycaena cupreus snowi (Edwards, 1881) & 22 \\
\hline Lycaena dione (Scudder, 1868) & 51 \\
\hline Lycaena dorcas arcticus (Ferris, 1977) & 1 \\
\hline Lycaena dorcas castro (Reakirt, 1866) & 37 \\
\hline Lycaena dorcas claytoni Brower, 1940 & 17 \\
\hline Lycaena dorcas dorcas Kirby, 1837 & 37 \\
\hline Lycaena dorcas florus (Edwards, 1884) & 24 \\
\hline Lycaena dospassosi McDunnough, 1940 & 17 \\
\hline Lycaena editha editha (Mead, 1878) & 43 \\
\hline Lycaena editha obscuramaculata Austin, 1989 & 2 \\
\hline Lycaena editha pseudonexa Emmel \& Pratt, 1998 & 65 \\
\hline Lycaena editha vurali Koçak, 1984 & 306 \\
\hline Lycaena epixanthe epixanthe (Boisduval \& Le Conte, 1835) & 46 \\
\hline Lycaena epixanthe michiganensis Rawson, 1948 & 13 \\
\hline Lycaena epixanthe phaedrus (Hall, 1924) & 13 \\
\hline Lycaena gorgon dorothea Emmel \& Pratt, 1998 & 75 \\
\hline Lycaena gorgon gorgon (Boisduval, 1852) & 78 \\
\hline Lycaena gorgon jacquelineae Emmel \& Pratt, 1998 & 65 \\
\hline Lycaena helloides (Boisduval, 1852) & 450 \\
\hline Lycaena hermes (Edwards, 1870) & 32 \\
\hline Lycaena heteronea undetermined sspp. & 43 \\
\hline Lycaena heteronea clara Edwards, 1877 & 2 \\
\hline Lycaena heteronea gravenotata Klots, 1930 & 28 \\
\hline Lycaena heteronea heteronea Boisduval, 1852 & 4 \\
\hline Lycaena heteronea klotsi Field, 1936 & 283 \\
\hline
\end{tabular}


Table 1 (continued).

Taxon

\# specimens

\begin{tabular}{|c|c|}
\hline Lycaena heteronea northi Emmel \& Pratt, 1998 & 60 \\
\hline Lycaena heteronea rava Austin, 1998 & 61 \\
\hline Lycaena heteronea submaculata Emmel \& Pratt, 1998 & 35 \\
\hline Lycaena hyllus (Cramer, 1775) & 58 \\
\hline Lycaena mariposa charlottensis (Holland, 1930) & 48 \\
\hline Lycaena mariposa mariposa (Reakirt, 1866) & 278 \\
\hline Lycaena mariposa penroseae Field, 1938 & 104 \\
\hline Lycaena nivalis bichroma Emmel \& Pratt, 1998 & 287 \\
\hline Lycaena nivalis browni Passos, 1938 & 151 \\
\hline Lycaena nivalis nivalis (Boisduval, 1869) & 37 \\
\hline Lycaena nivalis praetexta Austin, 1998 & 30 \\
\hline Lycaena nivalis warnermontana Emmel \& Pratt, 1998 & 31 \\
\hline Lycaena phlaeas alpestris Emmel \& Pratt, 1998 & 3 \\
\hline Lycaena phlaeas arethusa (Walley-Dod, 1907) & 4 \\
\hline Lycaena phlaeas feildeni (M'Lachlan, 1878) & 2 \\
\hline Lycaena phlaeas hypophlaeas (Boisduval, 1852) & 97 \\
\hline Lycaena rubidus duofascies Johnson \& Balogh, 1977 & 112 \\
\hline Lycaena rubidus ferrisi Johnson \& Balogh, 1977 & 2 \\
\hline Lycaena rubidus longi Johnson \& Balogh, 1977 & 18 \\
\hline Lycaena rubidus perkinsorum Johnson \& Balogh, 1977 & 51 \\
\hline Lycaena rubidus rubidus (Behr, 1866) & 58 \\
\hline Lycaena rubidus sirius (Edwards, 1871) & 135 \\
\hline Lycaena xanthoides nigromaculata Emmel \& Pratt, 1998 & 59 \\
\hline Lycaena xanthoides xanthoides (Boisduval, 1852) & 58 \\
\hline Ministrymon clytie (Edwards, 1877) & 23 \\
\hline Ministrymon leda (Edwards, 1882) & 51 \\
\hline Parrhasius m-album (Boisduval and Le Conte, 1833) & 31 \\
\hline Philotes sonorensis (Felder \& Felder, 1865) & 135 \\
\hline Philotiella leona Hammond \& McCorkle, 2000 & 146 \\
\hline Philotiella speciosa purisima Priestaf \& Emmel, 1998 & 4 \\
\hline Philotiella speciosa septentrionalis Austin, 1998 & 15 \\
\hline Philotiella speciosa speciosa (Edwards, 1877) & 66 \\
\hline Plebejus acmon (Westwood, 1852) & 604 \\
\hline Plebejus anna undetermined sspp. & 37 \\
\hline Plebejus anna anna (Edwards, 1861) & 118 \\
\hline Plebejus anna azureus (Emmel, Emmel \& Mattoon, 1998) & 19 \\
\hline
\end{tabular}


Table 1 (continued).

Taxon

\#specimens

\begin{tabular}{|c|c|}
\hline Plebejus anna ricei Cross, 1937 & 511 \\
\hline Plebejus anna vancouverensis (Guppy \& Shepard, 2001) & 8 \\
\hline Plebejus emigdionis (Grinnell, 1905) & 89 \\
\hline Plebejus glandon undetermined sspp. & 31 \\
\hline Plebejus glandon megalo McDunnough, 1927 & 83 \\
\hline Plebejus glandon rustica (Edwards, 1865) & 153 \\
\hline Plebejus icarioides undetermined sspp. & 37 \\
\hline Plebejus icarioides albihalos (Emmel, Emmel \& Mattoon, 1998) & 9 \\
\hline Plebejus icarioides atascadero (Emmel, Emmel \& Mattoon, 1998) & 1 \\
\hline Plebejus icarioides blackmorei Barnes \& McDunnough, 1919 & 42 \\
\hline Plebejus icarioides buchholzi Passos, 1938 & 21 \\
\hline Plebejus icarioides fenderi Macy, 1931 & 340 \\
\hline Plebejus icarioides fulla (Edwards, 1870) & 206 \\
\hline Plebejus icarioides helios (Edwards, 1871) & 175 \\
\hline Plebejus icarioides icarioides (Boisduval, 1852) & 54 \\
\hline Plebejus icarioides inyo (Emmel, Emmel \& Mattoon, 1998) & 8 \\
\hline Plebejus icarioides lycea (Edwards, 1864) & 41 \\
\hline Plebejus icarioides missionensis Hovanitz, 1937 & 26 \\
\hline Plebejus icarioides montis Blackmore, 1923 & 264 \\
\hline Plebejus icarioides pardalis (Behr, 1867) & 72 \\
\hline Plebejus icarioides pembina (Edwards, 1862) & 583 \\
\hline Plebejus icarioides pheres (Boisduval, 1852) & 12 \\
\hline Plebejus idas alaskensis Chermock, 1945 & 55 \\
\hline Plebejus idas atrapraetextus Field, 1939 & 165 \\
\hline Plebejus idas empetri Freeman, 1938 & 6 \\
\hline Plebejus idas longinus (Nabokov, 1949) & 18 \\
\hline Plebejus idas scudderii (Edwards, 1861) & 25 \\
\hline Plebejus idas sublivens (Nabokov, 1949) & 12 \\
\hline Plebejus lupini undetermined sspp. & 37 \\
\hline Plebejus lupini alpicola (Emmel, Emmel \& Mattoon, 1998) & 5 \\
\hline Plebejus lupini chlorina (Skinner, 1902) & 70 \\
\hline Plebejus lupini dedeckera (Emmel, Emmel \& Mattoon, 1998) & 4 \\
\hline Plebejus lupini goodpasturei (Austin, 1998) & 5 \\
\hline Plebejus lupini lutzi Passos, 1938 & 355 \\
\hline Plebejus lupini spangelatus (Burdick, 1942) & 27 \\
\hline Plebejus lupini texanus (Goodpasture, 1973) & 37 \\
\hline
\end{tabular}


Table 1 (continued).

Taxon

\#specimens

\begin{tabular}{|c|c|}
\hline Plebejus melissa undetermined sspp. & 44 \\
\hline Plebejus melissa annetta (Edwards, 1882) & 3 \\
\hline Plebejus melissa inyoensis (Nabokov, 1949) & 14 \\
\hline Plebejus melissa melissa (Edwards, 1873) & 408 \\
\hline Plebejus melissa samuelis (Nabokov, 1944) & 2 \\
\hline Plebejus neurona (Skinner, 1902) & 88 \\
\hline Plebejus optilete (Knoch, 1781) & 4 \\
\hline Plebejus podarce cilla (Behr, 1867) & 44 \\
\hline Plebejus podarce klamathensis (Emmel \& Emmel, 1998) & 116 \\
\hline Plebejus podarce podarce (Felder \& Felder, 1865) & 15 \\
\hline Plebejus saepiolus undetermined sspp & 864 \\
\hline Plebejus saepiolus amica (Edwards, 1863) & 56 \\
\hline Plebejus saepiolus hilda (Grinnell, 1907) & 24 \\
\hline Plebejus shasta undetermined sspp. & 21 \\
\hline Plebejus shasta minnehaha (Scudder, 1874) & 4 \\
\hline Plebejus shasta pallidissima (Austin, 1998) & 4 \\
\hline Plebejus shasta pitkinensis Ferris, 1976 & 14 \\
\hline Plebejus shasta shasta (Edwards, 1862) & 192 \\
\hline Rekoa marius (Lucas, 1857) & 1 \\
\hline Satyrium acadica (Edwards, 1862) & 64 \\
\hline Satyrium acadica montanensis (Watson \& Comstock, 1920) & 3 \\
\hline Satyrium alcestis alcestis & 22 \\
\hline Satyrium alcestis oslari & 2 \\
\hline Satyrium auretorum (Boisduval, 1852) & 79 \\
\hline Satyrium behrii undetermined sspp. & 40 \\
\hline Satyrium behrii behrii (Edwards, 1870) & 26 \\
\hline Satyrium behrii columbia (McDunnough, 1944) & 175 \\
\hline Satyrium behrii crossi (Field, 1938) & 52 \\
\hline Satyrium calanus undetermined sspp. & 127 \\
\hline Satyrium californica undetermined sspp & 410 \\
\hline Satyrium caryaevorus (McDunnough, 1942) & 28 \\
\hline Satyrium edwardsii undetermined sspp. & 65 \\
\hline Satyrium favonius autolycus (Edwards, 1871) & 90 \\
\hline Satyrium favonius favonius (Smith, 1797) & 40 \\
\hline Satyrium favonius ontario (Edwards, 1868) & 16 \\
\hline Satyrium fuliginosa (Edwards, 1861) & 18 \\
\hline
\end{tabular}


Table 1 (continued).

Taxon

\# specimens

\begin{tabular}{|c|c|}
\hline Satyrium fuliginosa tildeni Mattoon \& Austin, 1998 & 16 \\
\hline Satyrium ilavia (Beutenmüller, 1899) & 1 \\
\hline Satyrium kingi (Klots \& Clench, 1952) & 15 \\
\hline Satyrium liparops (LeConte, 1833) & 44 \\
\hline Satyrium liparops floridensis Gatrelle, 2001 & 1 \\
\hline Satyrium polingi organensis Ferris, 1980 & 2 \\
\hline Satyrium polingi polingi (Barnes \& Benjamin, 1926) & 17 \\
\hline Satyrium saepium (Boisduval, 1852) & 389 \\
\hline Satyrium semiluna semiluna Klots, 1930 & 230 \\
\hline Satyrium sylvinus undetermined sspp. & 257 \\
\hline Satyrium sylvinus desertorum (Grinnell, 1917) & 6 \\
\hline Satyrium sylvinus dryope (Edwards, 1870) & 35 \\
\hline Satyrium sylvinus itys (Edwards, 1882) & 2 \\
\hline Satyrium sylvinus megapallidum Austin, 1998 & 89 \\
\hline Satyrium tetra (Edwards, 1870$)$ & 113 \\
\hline Satyrium titus campus (Gatrelle, 2004) & 5 \\
\hline Satyrium titus immaculosus (Comstock, 1913) & 88 \\
\hline Satyrium titus occidentalis (Austin \& Emmel, 1998) & 1 \\
\hline Satyrium titus titus (Fabricius, 1793) & 15 \\
\hline Satyrium titus watsoni (Barnes \& Benjamin, 1926) & 4 \\
\hline Satyrium titus winteri (Gatrelle, 2004) & 49 \\
\hline Strymon acis (Drury, 1773) & 9 \\
\hline Strymon alea (Godman \& Salvin, 1887) & 9 \\
\hline Strymon avalona (Wright, 1905) & 4 \\
\hline Strymon bazochii bazochii (Godart, 1824) & 38 \\
\hline Strymon istapa (Reakirt, 1867) & 55 \\
\hline Strymon martialis (Herrich-Schäffer, 1864) & 15 \\
\hline Strymon melinus Hübner, 1818 & 470 \\
\hline Strymon melinus melinus Hübner, 1818 & 2 \\
\hline Strymon melinus setonia McDunnough, 1927 & 2 \\
\hline Strymon rufofusca (Hewitson, 1877) & 6 \\
\hline Tmolus echion (Linnaeus, 1767) & 6 \\
\hline Vaga blackburni (Tuely, 1878) & 28 \\
\hline Zizula cyna (Edwards, 1881) & 3 \\
\hline
\end{tabular}


Table 2.

Taxon

\# specimens

\begin{tabular}{|c|c|}
\hline Apodemia duryi (Edwards, 1882) & 1 \\
\hline Apodemia mejicanus mejicanus (Behr, 1865) & 47 \\
\hline Apodemia mejicanus pueblo Scott, 1998 & 6 \\
\hline Apodemia mormo autumnalis Austin, 1998 & 80 \\
\hline Apodemia mormo cythera Edwards, 1873 & 25 \\
\hline Apodemia mormo undetermined sspp. & 1 \\
\hline Apodemia mormo langei Comstock, 1939 & 65 \\
\hline Apodemia mormo mormo Felder, 1859 & 184 \\
\hline Apodemia mormo parva Austin, 1998 & 4 \\
\hline Apodemia nais Edwards, 1871 & 54 \\
\hline Apodemia palmerii arizona Austin, 1989 & 81 \\
\hline Apodemia palmerii palmerii Edwards, 1870 & 9 \\
\hline Apodemia virgulti davenporti Emmel, Emmel \& Pratt, 1998 & 13 \\
\hline Apodemia virgulti virgulti (Behr, 1865) & 130 \\
\hline Calephelis arizonensis McAlpine, 1971 & 40 \\
\hline Calephelis borealis (Grote \& Robinson, 1866) & 31 \\
\hline Calephelis freemani McAlpine, 1971 & 28 \\
\hline Calephelis muticum McAlpine, 1937 & 13 \\
\hline Calephelis nemesis Edwards, 1871 & 65 \\
\hline Calephelis perditalis perditalis Barnes \& McDunnough, 1918 & 89 \\
\hline Calephelis rawsoni McAlpine, 1939 & 2 \\
\hline Calephelis virginiensis Gray, 1832 & 19 \\
\hline Calephelis wrighti Holland, 1930 & 1 \\
\hline Caria ino melicerta Schaus, 1890 & 44 \\
\hline Emesis ares (Edwards, 1882) & 27 \\
\hline Emesis emesia Hewitson, 1867 & 1 \\
\hline Emesis zela cleis (Edwards, 1882) & 62 \\
\hline Lasaia sula peninsularis Clench, 1971 & 23 \\
\hline
\end{tabular}

Additional material from outside of North America is present, but was not digitized and catalogued at this time. 
Label data for these specimens can be accessed via online data portals that serve osac IPT data (e.g., www.gbif.org) or by direct download via the archived datasets stored in the supplemental files of this paper.

\section{Discussion:}

Today the Oregon State Arthropod Collection maintains a relatively comprehensive representation of North American Lycaenidae, built up over many decades through the contributions of at least 20 private collectors.

A total of 156 species of Lycaenidae have been recorded from North America and Hawaii north of Mexico as reported in Pelham (2008) catalogue. Of these, $149(96 \%)$ are represented in the collection at the time of this writing, although 16 of these are represented by specimens from outside of this geographic range (e.g. further to the south) and were not included in this dataset.

The majority of specimens is made up of taxa that occur in the Pacific Northwest and California, with generally good geographic coverage within these regions. In numerous cases (Table 1) subspecies were not always formally recognized in the database given uncertainties as to their validity and / or diagnosability. For example, while Euphilotes columbiae and Euphilotes ancilla are treated as valid species in Pelham (2008) there is some disagreement among lepidopterists as to their proper rank (e.g, the are often treated as subspecies of Euphilotes enoptes) and not all lepidopterists consider them valid. The OSAC does possess determined material corresponding to all of these nominative taxa, but at the time of this publication we opted to treat them as Euphilotes enoptes undetermined sspp. until we have more time to carefully review the taxonomy in greater detail.

Minor corrections and other small modifications made in the future to this dataset will be accomplished by issuing a new version and providing a file of modified records to the supplemental files. Major additions, such as new records, new data fields, new contributors or other significant modifications will be accomplished through the generation and release of new datasets.

\section{Acknowledgments}

The records published herein were generated with funds from NSF: DBI\#1601888: Digitization TCN: Collaborative Research: Lepidoptera of North America Network: Documenting Diversity in the Largest Clade of Herbivores.

\section{References}

Pelham JP (2008) A catalogue of the butterflies of the United States and Canada. Journal of Research on the Lepidoptera 40: 1-658

Seltmann, K. C. et al. (2017) LepNet: The Lepidoptera of North America Network. Zootaxa 4247 (1): 073-077. https:/ / doi.org/10.11646/zootaxa.4247.1.10.

Shepard J.H., C.J. Marshall, P.C. Hammond. 2018. North American Lepidoptera in the Oregon State Arthropod Collection: Hesperiidae, Pieridae, Papilionidae. Catalog: Oregon State Arthropod Collection 2(2): 1-2 and supplement file. doi: http://dx.doi.org/10.5399/osu/cat osac.2.2.4480 